\title{
ON SYMMETRY OF BANACH JORDAN ALGEBRAS
}

\author{
B. AUPETIT ${ }^{1}$ AND M. A. YOUNGSON
}

\begin{abstract}
Using a very simple subharmonic argument we prove that a Banach Jordan algebra is Hermitian if and only if the sum of two positive elements is positive. We apply this result to give a characterization of Banach Jordan algebras with involution which are $\mathrm{JB}^{*}$-algebras for an equivalent norm.
\end{abstract}

If $B$ is a complex Banach algebra with involution, the Shirali-Ford theorem states that every selfadjoint element of $B$ has real spectrum if and only if $x^{*} x$ has positive spectrum for every $x$ in $A$. In particular this theorem implies that such an algebra has many Hilbert space representations. The corresponding problem in Banach Jordan algebras is also important as if a Hermitian algebra is symmetric it. is possible to make use of the representation theory for JB-algebras given by Alfsen, Shultz and Størmer in [1]. A solution to this problem was given by Behncke in [5] but the argument he used, while containing a nice idea, is rather long. The aim of this paper is to give a simple proof of Behncke's result using a subharmonic argument and to give an application to the characterization of JB*-algebras.

Instead of working with real Banach Jordan algebras throughout this paper we shall let $A$ denote a complex unital Banach Jordan algebra with involution and $S$ shall denote the real linear subspace of selfadjoint elements of $A$. As for Banach algebras, if $a \in A$ one can define the spectrum and spectral radius of $a$; these will be denoted by $\operatorname{Sp}(a)$ and $\rho(a)$, respectively. Some standard properties of $\operatorname{Sp}(a)$ and $\rho(a)$ are given in [4 and 8], for example. Each $a \in A$ induces a linear operator $U_{a}$ on $A$ defined by $U_{a}(b)=2 a(a b)-b\left(a^{2}\right)$. It is shown in [7] that $a$ is invertible if and only if $U_{a}$ is invertible and further that $a$ and $b$ are invertible if and only if $U_{a} b$ is invertible.

We recall from [4] that if $D$ is a domain in the complex numbers $\mathbf{C}$ and $f: D \rightarrow A$ is an analytic function then $\lambda \rightarrow \rho(f(\lambda))$ is a subharmonic function.

Lemma. Let $D$ be a domain in $\mathbf{C}$ containing $[0,1]$ and let $f: D \rightarrow A$ be an analytic function. If $a<b<c<d$ are real numbers such that $\operatorname{Sp}(f(t)) \subset[a, b] \cup[c, d]$ for all $t \in[0,1]$ and $\operatorname{Sp}(f(0)) \subset[a, b]$, then $\operatorname{Sp}(f(t)) \subset[a, b]$ for all $t \in[0,1]$.

Proof. We may assume that $a>0$. Let $E=\{t \in[0,1]: \operatorname{Sp}(f(t)) \subset[a, b]\}$. We first show that $E$ is open in $[0,1]$. We assume conversely that there exist $t_{0} \in E$ and a sequence $\left(t_{n}\right)$ in $[0,1]$ converging to $t_{0}$ with $t_{n} \notin E$ for all $n \in \mathbf{N}$. Hence there exists $\alpha_{n} \in \operatorname{Sp}\left(f\left(t_{n}\right)\right) \cap[c, d]$ for all $n \in \mathbf{N}$. By passing to a subsequence if

Received by the editors October 1, 1982.

1980 Mathematics Subject Classification. Primary 46H05; Secondary 17C59.

Key words and phrases. Banach Jordan algebra, spectrum, spectral radius, subharmonic function, $\mathrm{JB}^{*}$-algebra.

${ }^{1}$ Supported by a Senior Fellowship of the British Science and Engineering Research Council at the University of Stirling, Scotland. 
necessary, we may assume that $\left(\alpha_{n}\right)$ converges to some $\alpha \in[c, d]$. Thus $\alpha-f\left(t_{0}\right)$ is an invertible element which is the limit of a sequence $\left(\alpha_{n}-f\left(t_{n}\right)\right)$ of noninvertible elements. This contradicts the fact that the set of invertible elements is open. Hence $E$ is open in $[0,1]$. Let $\gamma=\operatorname{Sup}\{r \in[0,1],[0, r] \subset E\}$. As $E$ is open, $0<\gamma \leq 1$. Further $[0, \gamma) \subset E$ and because $\lambda \rightarrow \rho(f(\lambda))$ is subharmonic and $[0, \gamma)$ is nonthin at $\gamma$ (see [2, pp. 170-171]) we have

$$
\rho(f(\gamma))=\lim \sup \{\rho(f(r)): r<\gamma\} \leq b .
$$

hence $\gamma \in E$ and so as $E$ is open in $[0,1]$, we have $\gamma=1$ and the result follows.

Using a similar argument (see [2, p. 34]) with subharmonicity of $\lambda \rightarrow \rho(f(\lambda))$ it is possible to prove the analogue of Newburgh's theorem [2, p. 8, Theorem 4] for Banach Jordan algebras which of course implies this lemma. We can also use directly the stronger result on the disintegration of analytic multivalued functions given in [3, Theorem 3.14].

We now prove Behncke's result. We follow the same method of proof as he does initially. but by our introduction of analytic functions and the use of the lemma we avoid his rather complicated calculations.

THEOREM. The following are equivalent:

(i) $A$ is Hermitian, that is, if $h \in S$ then $\operatorname{Sp}(h) \subset \mathbf{R}$;

(ii) if $h, k \in S$ have positive spectra then so has $h+k$;

(iii) $x^{*} x$ has positive spectrum for all $x$ in $A$.

ProOF. The implications of (ii) implies (iii) and (iii) implies (i) are easy and so we only show that (i) implies (ii). Suppose conversely that there exist $h, k \geq 0$ such that $h+k$ does not have positive spectrum. Then there exists $\gamma>0$ such that $h+k+\gamma$ is not invertible. Since $h+\gamma / 2$ and $k+\gamma / 2$ are positive and invertible we can find invertible $a$ and $b$ in $A$ such that $a^{2}=h+\gamma / 2$ and $b^{2}=k+\gamma / 2$ and the spectrum of $a$ and $b$ is positive. Now $U_{a}\left(1+U_{a^{-1}}\left(b^{2}\right)\right)=a^{2}+b^{2}$ is not invertible in $A$ while $a$ is invertible. Hence $1+U_{a^{-1}}\left(b^{2}\right)$ is not invertible which means that $-1 \in \operatorname{Sp}\left(U_{a^{-1}}\left(b^{2}\right)\right)$. Let $f: \mathbf{C} \rightarrow A$ be the analytic function defined by $f(\lambda)=U_{a^{-1}}\left((1-\lambda) b^{2}+\lambda\right)$. As $(1-\lambda) b^{2}+\lambda$ is invertible for $0 \leq \lambda \leq 1$ the upper semicontinuous functions $\lambda \rightarrow \rho(f(\lambda))$ and $\lambda \rightarrow \rho\left(f(\lambda)^{-1}\right)$ are bounded on $[0,1]$ and so there exist $0<\alpha<1<\beta$ such that $\operatorname{Sp}(f(t)) \subset[-\beta,-\alpha] \cup[\alpha, \beta]$ for $t \in[0,1]$. However $\operatorname{Sp} f(1)=\operatorname{Sp}\left(a^{-2}\right) \subset[\alpha, \beta]$ while $-1 \in \operatorname{Sp}(f(0))$ which contradicts the result of the Lemma.

It follows easily, as indicated in [5], that if $A$ is Hermitian and $h, k \in S$ then $\rho(h+k) \leq \rho(h)+\rho(k)$ and $\rho(h k) \leq \rho(h) \rho(k)$. Moreover the set of positive elements is closed in the set of selfadjoint elements. Using these results we can improve the characterization of $\mathrm{JB}^{*}$-algebras in an equivalent norm given in [8]. We recall that $A$ is a $\mathrm{JB}^{*}$-algebra if for every $x$ in $A$ we have $\left\|U_{x}\left(x^{*}\right)\right\|=\|x\|^{3}$. It is easy to check that if $A$ is a $\mathrm{JB}^{*}$-algebra and $h \in S$ then $\|\exp (i h)\|=1$. This establishes part of the following Corollary.

COROLlary. $A$ is a $\mathrm{JB}^{*}$-algebra in an equivalent norm if and only if there exists a constnt $C \geq 1$ such that $\|\exp (i h)\| \leq C$ for all $h \in S$.

ProOF. By the remark above we need only show that $A$ is $\mathrm{JB}^{*}$-equivalent if the given condition holds. If $h \in S$ then $\|\exp (i n h)\| \leq C$ for all $n \in \mathbf{Z}$. Thus 
$\rho(\exp (i h))=\rho(\exp (-i h))=1$ and so $\operatorname{Sp}(h) \subset \mathbf{R}$. By our theorem and the preceding remarks $\rho$ is subadditive on $S$. We now show that the McCrimmon radical of $A$ is zero. If $x \in \operatorname{Rad} A$ then $h=\left(x+x^{*}\right) / 2$ and $k=\left(x-x^{*}\right) / 2 i$ are also in $\operatorname{Rad} A$. Hence $\rho(h)=\rho(k)=0$. However by an argument similar to part of the proof of [8, Theorem 11], the closed subalgebra generated by $h$ and 1 has an equivalent algebra norm $\|||||$ such that $\rho(h)=\|\||||| \mid$ and $\left\|\left|h\left\|\mid \geq C^{-2}\right\| h \|\right.\right.$. Hence $h=0$ and similarly $k=0$ and so $x=0$. Thus by [4, Corollary 3$]$, the involution is continuous and the desired result holds by [8, Theorem 11].

\section{REFERENCES}

1. E. M. Alfsen, F. W. Shultz and E. Størmer, A Gelfand-Neumark theorem for Jordan algebras, Adv. in Math. 28 (1978), 11-56.

2. B. Aupetit, Propriétés spectrales des algèbres de Banach, Lecture Notes in Math., vol. 735, Springer-Verlag, Heidelberg, 1979.

3. __ Analytic multivalued functions in Banach algebras and uniform algebras, Adv. in Math. 44 (1982), 18-60.

$\dot{4}$. The uniqueness of the complete norm topology in Banach algebras and Banach Jordan algebras, J. Funct. Anal. 47 (1982), 1-6.

5. H. Behncke, Hermitian Jordan Banach algebras, J. London Math. Soc. (2) 20 (1979), 327-333.

6. F. F. Bonsall and J. Duncan, Complete normed algebras, Springer-Verlag, Heidelberg, 1973.

7. N. Jacobson, Structure and representations of Jordan algebras, Amer. Math. Soc. Colloq. Publ., vol. 39, Amer. Math. Soc., Providence, R.I., 1968.

8. M. A. Youngson, Equivalent norms on Banach Jordan algebras, Math. Proc. Cambridge Philos. Soc. 86 (1979), 261-269.

DÉPaRtement de Mathématiques, UniVERsité laVal, QUÉBEC, CANADA G1K 7P4

Department of MAThematics, Heriot-Watt University, Edinburgh, SCOTLAND EH14 4A5 